\title{
Implicaciones de la precipitación sobre la evolución del tamaño corporal y distancia interaxilar en el complejo Aspidoscelis gularis (Squamata: Teiidae)
}

\author{
Carlos Pérez-Almazán ${ }^{1 *}$, Norma L. Manríquez-Morán², Miguel A. Balderas-Plata ${ }^{3}$, \\ Xanat Antonio Némiga ${ }^{3}$ \& Saúl López-Alcaide ${ }^{2}$ \\ 1. Facultad de Ingeniería. Universidad Autónoma del Estado de México, Instituto Literario 100, Colonia Centro, Toluca, \\ Estado de México, México, C. P. 50000; cpa52013@gmail.com \\ 2. Laboratorio de Sistemática Molecular. Universidad Autónoma del Estado de Hidalgo, Ciudad del Conocimiento, \\ Carretera Pachuca- Tulancingo Km. 4.5, Col. Carboneras, Mineral de la Reforma, Hidalgo, C. P. 42184; \\ nrm292@gmail.com,sla@st.ib.unam.mx \\ 3. Facultad de Geografia, Laboratorio de Suelos y Laboratorio de Sistemas de Información Geográfica. Universidad \\ Autónoma del Estado de México, Instituto Literario 100, Colonia Centro, Toluca, Estado de México, México, C. P. \\ 50000; mplata@colpos.mx, xanynemiga@rocketmail.com
}

Recibido 07-IV-2016. Corregido 12-XII-2016. Aceptado 16-I-2017.

\begin{abstract}
Rainfall implications on body size evolution of Aspidoscelis gularis (Squamata: Teiidae). Life history traits are highly variable attributes that maximize organisms's fitness. The relationship of weight and body size with environmental changes and habitat heterogeneity has been documented in previous reports; and size and body shapes are both considered life history attributes that are associated with rainfall, that boost available resources in the environment. While in Aspidoscelis genus, clutch size and relative mass are mainly associated with latitude and altitude, in Aspidoscelis gularis, winter rainfall favors two reproductive seasons, which may determine season variable clutch size. With the aim to study this, samplings were undertaken from May-July 2013, and May-September 2015. A total of 65 individuals lizards of the Southeast clade were obtained, and body length and interaxilar distance measurements were taken; furthermore, hepatic tissue samples were taken for DNA extraction, which allowed us to analyze phylogenetic relationships through a Bayesian Inference analysis, and subsequently, to apply Phylogenetic Comparative Methods (like phylogenetic signal, phylogenetically independent contrasts and reconstruction of ancestral character). Our results showed that there is a low phylogenetic signal regarding body size and shape, while the phylogenetically independent contrasts and reconstruction of ancestral characters suggest that small body sizes are associated to locations with highest rainfall. This can be associated to an establishment of an early sexual maturity, which reflects the maximum size of adults. Furthermore, according to an ANOVA and ANCOVA, there were statistically significant differences in body size and shape respectively, which promote a system for sexual competition for males and a system for fertility in females. These results were important to determine the effect of rainfall on some life history traits, pointing out that lizards of the Southeast clade, belonging to the A. gularis complex were able to face different selection pressures, determined by the environment. Rev. Biol. Trop. 65 (2): 725-733. Epub 2017 June 01.
\end{abstract}

Key words: Phylogenetic Comparative Method, phylogenetical signal, phylogenetical independent contrast, life histories, evolutionary history.

Existen diversos factores que influyen sobre la expresión de los atributos de historia de vida, y en consecuencia, sobre la adecuación de los organismos (Blueweiss et al., 1978; Stearns, 1992). Su variación y heredabilidad son fundamentales para producir fenotipos con potencial adaptivo bajo selección natural (Stearns, 1976; Iraeta, Salvador \& Díaz, 2012). Se ha documentado la relación y efectos del peso y el tamaño corporal sobre algunos atributos de historia de vida (frecuencia reproductora, tamaño y masa relativa de la camada o nidada) 
(Angilleta, Oufiero \& Leaché, 2006; Brandt \& Navas, 2011), los cuales a menudo están influidos por variaciones ambientales y la heterogeneidad del hábitat (Mesquita, Gomes-Faria, Rinaldi-Colli, Vitt \& Pianka, 2016). El tamaño y la forma corporal (capacidad abdominal) se pueden considerar como un atributo de historia de vida en sí mismo, ya que su variación tiene efectos directos sobre la adecuación. La variación de estos atributos ha sido documentada como producto del efecto de la precipitación, distribución y estructura del hábitat (Brandt \& Navas, 2011; Grizante, Brandt \& Kohlsdorf, 2012; Pincheira-Donoso \& Meiri, 2013).

La precipitación, por ejemplo, tiene efectos sobre la disponibilidad de recursos en el ambiente, lo que en conjunto promueve la variación en los atributos de historia de vida (Michael et al., 2014; Meiri, Yom-To \& Geffen, 2007). Por ejemplo, la variación en la precipitación anual y en los recursos disponibles promueve una disminución en el tamaño de los cuerpos grasos en Urosaurus ornatus, lo que afectó el esfuerzo reproductor (Ballinger, 1977). En la subfamilia Tropidurinae, la precipitación favoreció un mayor tamaño corporal de la hembra, comparado con los machos, con una correlación positiva del tamaño corporal y el tamaño de nidada (Brandt \& Navas, 2011). Además, es importante considerar que madurez sexual está correlacionada con la talla máxima de adultos (Stearns, 1992), por lo que los recursos disponibles pueden promover un efecto directo sobre la talla a la cual la madurez puede ser alcanzada. Por ejemplo, en especies con hábitos de forrajeo activo, la adquisición de recursos es esencial para alcanzar una madurez sexual temprana y con ello reducir los costos asociados a la reproducción (Shine, 1980). Por otro lado, se ha descrito que la forma corporal como la distancia interaxilar en Aspidoscelis costata puede ser un atributo seleccionado a través del dimorfismo sexual por fecundidad, porque favorece un aumento significativo en el tamaño de nidada, con respecto a otros taxones del género (Aguilar-Moreno et al., 2010; Roitberg et al., 2015).
En general, los integrantes del género Aspidoscelis, poseen características ecológicas, conductuales, fisiológicas y morfológicas similares, con modo de forrajeo activo, preferencia por hábitats abiertos, con tácticas de escape altamente especializadas para evadir depredadores (Vitt \& Breitenbach, 1993; Pianka \& Vitt, 2003). Sin embargo, en el género Aspidoscelis, debido a la gran variación y a los factores de los cuales dependen, las historias de vida son complicadas de evaluar e inferir; por lo tanto, es importante abordar su estudio desde un contexto evolutivo mediante métodos filogenéticos comparados, para eliminar el efecto de la no-independencia estadística entre taxones cercanamente relacionados (Felsestein, 1985, Harvey \& Pagel, 1991). De esta manera, los métodos filogenéticos comparados permiten analizar diversos tipos de información (morfológica, fisiológica y ecológica) que con respecto a información filogenética pueden determinar de manera histórica la evolución (inercia filogenética) y adaptación de un estado de carácter (características de historia de vida), en respuesta a diferentes presiones de selección (Benabib, 2009; Zamora-Abrego, Manríquez-Morán, Ortíz-Yusty \& Ortega-León, 2013).

Las lagartijas del género Aspidoscelis son ovíparas, habitan en zonas tropicales y templadas y tienen modo de forrajeo activo, además presentan un intervalo de 1.4-5.6 huevos ( $A$. tigris-A. sacki, respectivamente). Dentro del grupo Aspidoscelis sexlineata se encuentran los integrantes del complejo A. gularis los cuales, en lo general, se encuentran asociados a la vegetación característica del desierto chihuahuense, y están distribuidos a través del Altiplano Mexicano hasta la Ciudad de México (PérezAlmazán, Balderas-Plata, Manríquez-Morán, Madrigal-Uribe \& Antonio-Némiga, 2014).

De acuerdo con un análisis filogenético reciente, para el complejo Aspidoscelis gularis, se lograron identificar tres clados principales (Chávez-García, 2010), donde sólo el clado sureste distribuido en Hidalgo, San Luís Potosí, Guanajuato, Querétaro y la Ciudad de México, es un grupo natural o monofilético, lo cual cumple con los supuestos para aplicar 
el método comparado filogenético. Por lo tanto, en este trabajo se evaluó la relación de la precipitación sobre el tamaño corporal y la distancia interaxilar, y se discuten las posibles consecuencias que tiene esta relación sobre atributos coadaptados reproductores, como frecuencia reproductora y el tamaño de nidada, los cuales pueden presentar variación interespecifica. Para esto, se obtuvieron los contrastes filogenéticamente independientes, para evaluar la relación de la precipitación sobre los atributos morfológicos considerados, así como la señal filogenética y la reconstrucción del carácter ancestral para estos atributos, tomando como referencia los taxones que integran el clado sureste del complejo A. gularis.

\section{MATERIALES Y MÉTODOS}

Se realizaron colectas a través de la distribución del clado sureste del complejo Aspidoscelis gularis durante mayo y julio 2013, y mayo y septiembre 2015 (DGVS-SEMARNAT_FAUT- 0243) (Cuadro 1). Se obtuvieron un total de 65 individuos (32 hembras y 33 machos, $\bar{X}=9.28$ individuos por localidad) los cuales fueron transportados, mantenidos y sacrificados en laboratorio, de acuerdo a la NOM-062-ZOO-1999. Se realizaron medidas morfométricas como longitud hocico-cloaca (LHC, mm) y distancia interaxilar (distancia entre miembros anteriores y posteriores, $\mathrm{mm}$ ) y se obtuvo tejido hepático. Todos los individuos colectados fueron disecados con formol al $10 \%$, y preservados en alcohol al $70 \%$, y se mantienen en la colección de anfibios y reptiles del Centro de Investigaciones Biológicas de la Universidad Autónoma del Estado de Hidalgo.

Extracción de ADN: Para obtener las relaciones filogenéticas ad hoc se extrajo ADN mediante la técnica de acetato de amonio (Fetzner, 1999). Éste es un método que fue propuesto para extraer $\mathrm{ADN}$ de la muda de reptiles, pero funciona para extraer el ADN genómico de diversos tejidos de animales, dejando el ADN con una pureza superior con respecto a otros métodos (Fetzner, 1999).

Amplificación de fragmentos del gen mitocondrial ND2: Se amplificó el gen mitocondrial que codifica para subunidad II de la NADH deshidrogenasa (ND2), mediante el método de la Reacción en Cadena de la Polimerasa (PCR) descrito por Palumbi (1996). Las amplificaciones se realizaron con un volumen final de $25 \mu \mathrm{L}(1.0 \mu \mathrm{L}$ de DNA, $0.2 \mu \mathrm{L}$ de cada primer, $1.0 \mu \mathrm{L}$ de dNTPs, 1.2 de $\mathrm{MgCl} 2$, $1.8 \mu \mathrm{L}$ de amortiguador, $0.15 \mu \mathrm{L}$ de Taq polimerasa y $19.5 \mu \mathrm{L}$ de $\mathrm{dH}_{2} \mathrm{O}$ ) (Espinosa, 2007). Los productos de PCR se visualizaron en geles de agarosa al $1 \%$ mediante electroforesis, y se purificaron a través de la técnica precipitación con polietilen-glicol (PEG) que elimina

CUADRO 1

Localidades consideras, identificadores y promedio anual precipitación de 6 años

TABLE 1

Considered localities, identifiers and mean annual precipitation of 6 years

\begin{tabular}{lcc}
\multicolumn{1}{c}{ Localidad } & Identificador & Precipitación \\
Mezquititlán, Hidalgo & SFDG144 & 72.456 \\
Mezquititlán, Hidalgo & SFDG146 & 72.456 \\
Jacala, Hidalgo & AMH673 & 60.425 \\
Zimapán, Hidalgo & JCHZ011 & 35.842 \\
Jalpan, Querétaro & OIGS201 & 37.677 \\
Jalpan, Querétaro & OIGS202 & 37.677 \\
El Rosario, Querétaro & CPA045 & 52.101 \\
Jerónimo de Aráceo, Guanajuato & CPA048 & 63.331 \\
Estación de la Cruz, Tamaulipas & CPA017 & 60.354 \\
\hline
\end{tabular}


residuos de los compuestos de la reacción de amplificación.

Inferencia filogenética y método comparativo filogenético: Para analizar la información a través de los métodos filogenéticos comparados propuestos, se obtuvieron las relaciones filogenéticas de individuos pertenecientes al clado sureste del complejo Aspidoscelis gularis (Fig. 1). De esta manera, se obtuvo el modelo de sustitución de nucleótidos HYK de acuerdo al criterio de inferencia bayesiana en MEGA v.6.06 (Tamura, Stecher, Peterson, Filipski \& Kumar, 2013). El análisis bayesiano consistió de dos corridas simultáneas, utilizando cuatro cadenas de Markov por 50 millones de generaciones, y se muestreó cada 1000 generaciones; para el resto de los parámetros se utilizaron los valores preestablecidos.

Señal filogenética y reconstrucción del carácter ancestral: Se utilizó la información filogenética obtenida para estimar la señal filogenética del tamaño corporal (para todos los taxones, para las hembras y machos), y la distancia interaxilar de hembras de las nueve poblaciones consideradas de los taxones del clado sureste del complejo Aspidoscelis gularis (Cuadro 1), asumiendo para estos estados carácter un modelo de evolución Browniano.

Los análisis se realizaron con el paquete "picante" para R (R Development Core Team, 2011) utilizando la matriz de caracteres morfológicos asociados al árbol filogenético y se calculó el valor de K (Bloomberg, Garland \& Ives, 2003). Un valor de $\mathrm{K}$ igual a 1 indica que el rasgo presenta exactamente la cantidad de señal predicha para una filogenia dada, asumiendo un modelo de evolución Browniano, mientras que valores menores que 1 significan menos señal que la esperada. Posteriormente, con el paquete "phytools" para R se realizó la reconstrucción del carácter ancestral a través

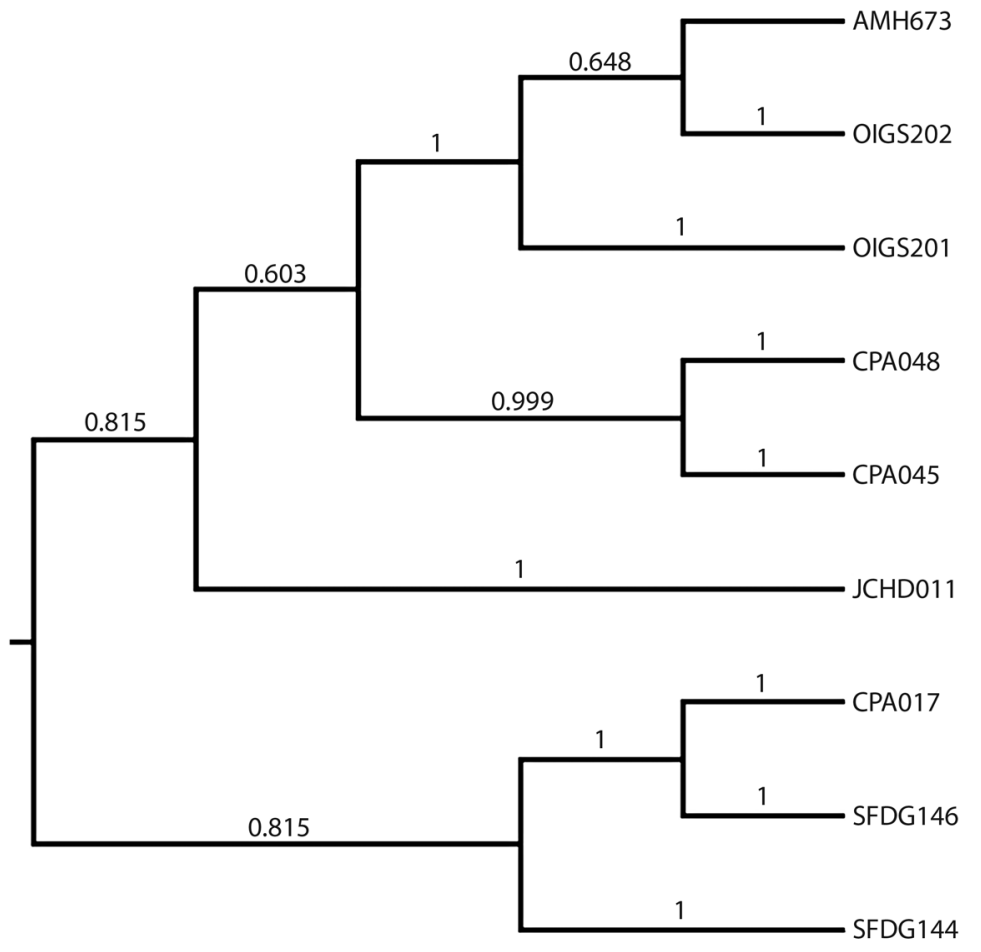

Fig. 1. Relaciones filogenéticas del clado sureste del complejo A. gularis.

Fig. 1. Phylogenetic relationships of Southeast clade of $A$. gularis complex. 
de un criterio de máxima verosimilitud, para inferir el patrón evolutivo del tamaño corporal.

Contrastes filogenéticamente independientes: Debido a la estructura jerárquica que presentan los taxones en función de sus relaciones filogenéticas, los datos obtenidos de las mismas no pueden considerarse independientes (Harvey \& Pagel, 1991; Martins, 1996). Para independizar los caracteres del efecto del ancestro común de los taxones terminales a evaluar, se obtuvieron $\mathrm{n}-1$ contrastes filogenéticamente independientes (Felsentein, 1985), utilizando la hipótesis filogenética ad hoc (Fig. 1). Posteriormente, se realizaron correlaciones simples y se evaluó la relación entre el tamaño corporal (de todos los individuos de las poblaciones utilizadas, así como de las hembras y machos), usando el módulo PDAP: PDTREE (Midford, Garland \& Maddison, 2010) en el programa MESQUITE v.2.75 (Madison \& Madison, 2009).

Además, para describir estadísticamente la variación en el tamaño corporal y la distancia interaxilar, en Statgraphics Centurion v.17.01 se realizó un análisis de varianza (ANOVA) para comparar el tamaño corporal entre poblaciones y entre sexos, y se llevó a cabo un análisis de covarianza (ANCOVA) para comparar la distancia interaxilar entre sexos eliminando el efecto del tamaño corporal.

Obtención y análisis de variables ambientales: Para obtener los datos ambientales, se obtuvieron datos históricos de precipitación de la Red de Estaciones del Instituto Nacional de Investigaciones Forestales, Agrícolas y Pecuarias (INIFAP), de los cuales se obtuvo el promedio de precipitación anual (mm) dentro del intervalo de 2010-2015 para cada localidad (Cuadro 1). Para esto se tomó en cuenta las estaciones climáticas más cercanas a cada zona con registros históricos. Los datos fueron analizados con un ANOVA para determinar las diferencias significativas entre años y entre meses de los años considerados.

\section{RESULTADOS}

Señal filogenética y contrastes filogenéticamente independientes: Para la señal filogenética, el valor de $\mathrm{K}$ indicó que ninguna de las variables morfológicas es independiente de la filogenia dentro del clado sureste (Cuadro 2). Las correlaciones simples efectuadas por medio de contrastes independientes filogenéticamente, indicaron que no existen relaciones significativas entre el tamaño corporal y la precipitación en las poblaciones del clado sureste del complejo A. gularis, tanto para machos como para hembras (Cuadro 2). No obstante, a través de la reconstrucción ancestral del carácter, se pudo inferir el patrón evolutivo del tamaño corporal en el grupo y su relación con la precipitación: las hembras con tamaños corporales menores se encuentran asociadas a localidades con mayor precipitación (Fig. 2; Cuadro 1). De acuerdo con el promedio anual, la localidad con mayor precipitación es Mezquititlán en Hidalgo y la menor es Jalpan de Serra en Querétaro (Cuadro 1); ambas localidades pertenecen a la distribución más sureña del desierto chihuahuense.

Comparación entre poblaciones: $\mathrm{Se}$ comparó el tamaño corporal entre sexos de todos los grupos que integran el clado sureste, donde de acuerdo con un ANOVA, existieron diferencias estadísticamente significativas, y los machos resultaron más grandes que las hembras (Cuadro 2). Además, de acuerdo a un ANCOVA, que comparó la distancia interaxilar entre sexos con el tamaño corporal como covariable, y se encontró que la distancia interaxilar fue más grande en hembras que en machos (Cuadro 2).

\section{DISCUSIÓN}

De acuerdo con la señal filogenética, las características consideradas (tamaño corporal y distancia interaxilar), no son dependientes de la filogenia por lo que su evolución está sujeta 


\section{CUADRO 2}

Señal filogenética y contrastes filogenéticamente independientes de características morfológicas de acuerdo a la precipitación. Y análisis de varianza y covarianza de características morfológicas

TABLE 2

Phylogenetical signal and phylogenetic independent contrast of morphological traits according to precipitation. And variance and covariance analysis of morphological traits

\begin{tabular}{|c|c|c|}
\hline Señal filogenética & $\mathrm{K}$ & $\mathrm{P}$ \\
\hline TC todos los grupos & 0.556 & 0.034 \\
\hline $\mathrm{TC}+$ & 0.346 & 0.851 \\
\hline $\mathrm{TC} \hat{0}$ & 0.442 & 0.615 \\
\hline DIA $q$ & 0.396 & 0.722 \\
\hline Contrastes independientes & $\mathrm{r}$ & $P$ \\
\hline Precipitación vs TC todos & 0.112 & 0.198 \\
\hline Precipitación vs TC $q$ & 0.313 & 0.067 \\
\hline Precipitación vs TC $\widehat{\partial}$ & 0.233 & 0.105 \\
\hline ANOVA & $\bar{X}(\mathrm{DE})$ & $\mathrm{P}$ \\
\hline TC entre $\delta^{\lambda}+$ & ô $81.42 \pm 12.92 ;$ ㅇ $70.09 \pm 9.11$ & 0.0001 \\
\hline Precipitación anual & (EE) & $\mathrm{P}$ \\
\hline Entre años & 4.09 & 0.7675 \\
\hline Entre meses & 5.59 & 0.0001 \\
\hline ANCOVA & $\bar{X}(\mathrm{EE})$ & $\mathrm{P}$ \\
\hline DIA entre $\delta \circ$ & o $35.00 \pm 0.32 ;$ q $36.00 \pm 0.33$ & $0.0469 ; \mathrm{TC}=00001$ \\
\hline TC hembras vs DIA $q$ & 0.878 & 0.001 \\
\hline
\end{tabular}

$\mathrm{TC}=$ tamaño corporal $(\mathrm{mm}) ; \mathrm{DIA}=$ distancia interaxilar $(\mathrm{mm}) ; \hat{\delta}=$ machos; $q=$ hembras.

$\overline{\mathrm{X}}=$ promedio; $(\mathrm{DE})=$ Desviación Estándar; $(\mathrm{EE})=$ Error Estándar.

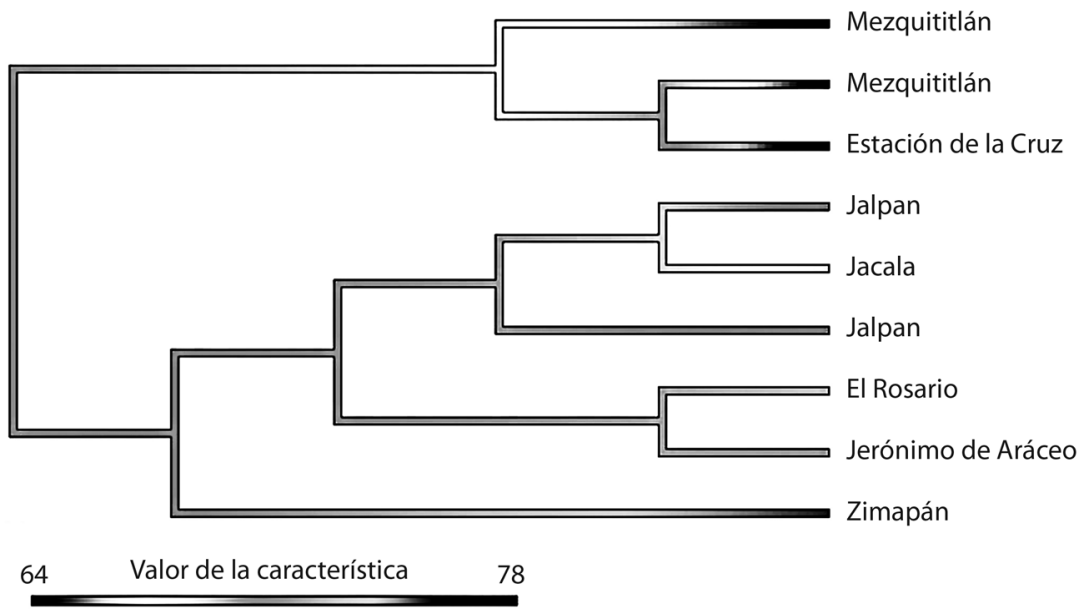

Fig. 2. Reconstrucción del carácter ancestral del tamaño corporal de hembras del clado sureste y la media de precipitación anual por localidad.

Fig. 2. Ancestral character reconstruction of the body size females of the Southeast clade and mean annual precipitation by locality. 
a otros procesos de selección. Por lo tanto, con respecto a los contrastes filogenéticamente independientes, se demostró que las lagartijas del clado sureste del complejo Aspidoscelis gularis, presentan una correlación negativa entre el tamaño corporal y la precipitación, lo que sugiere que los tamaños corporales pequeños se encuentran asociados a localidades con mayor cantidad de precipitación. Así mismo, la reconstrucción del carácter ancestral del tamaño corporal demostró que los tamaños corporales grandes han evolucionado en respuesta a la disminución de la precipitación.

La precipitación tiene una importante influencia y relación sobre la cantidad de recursos disponibles y de asignación, lo cual puede favorecer un espectro amplio y variable de estrategias de historia de vida, lo cual es un factor que maximiza la adecuación en un hábitat determinado. Por ejemplo, las hembras de Aspidoscelis tigris tienden a producir nidadas más grandes en años donde la precipitación fue mayor que el promedio registrado, debido al aumento en la cantidad de alimento disponible (Pianka, 1970; Pianka \& Vitt, 2003). En Uta stansburiana el promedio de la frecuencia de nidadas para todos los grupos de hembras estudiado fue positivamente relacionado a la lluvia total de invierno, particularmente durante noviembre-febrero donde las primeras nidadas son más grandes en comparación con las posteriores (Turner, Medica \& Smith, 1974; Vitt \& Breitenbach, 1993; Pianka \&Vitt, 2003). Algo similar ocurre en hembras de Sceloporus undulatus, las cuales produjeron dos puestas por estación reproductora, y en donde los huevos de la primera nidada (en invierno) fueron relativamente más pequeños y el tamaño de nidada grande, contrario a lo que sucede en la segunda puesta (en verano); este resultado está relacionado directamente con los recursos disponibles (Pianka \& Vitt, 2003). Además, es importante considerar los estudios realizados por Vitt y Breitenbach (1993) con taxones del complejo A. gularis al norte del área de distribución, donde se encontró evidencia que la frecuencia reproductora está asociada a las precipitaciones que van de noviembre a enero, lo que promueve dos puestas en un intervalo de doce meses. Lo anterior sugiere que estos individuos reproductivos deben alcanzar su madurez sexual tempranamente, ya que según Stearns (1992), los tamaños corporales de los adultos están correlacionados positivamente con el establecimiento de ésta.

Sumado a lo anterior, con respecto a los análisis de varianza y co-varianza en el clado sureste se identificaron diferencias estadísticamente significativas en el tamaño corporal y la distancia interaxilar. Lo cual puede sugerir que los machos se encuentran en un sistema de competencia de acuerdo al tamaño corporal y a las hembras en un sistema de selección para la fecundidad de acuerdo a la distancia interaxilar (Shine, 1989; Cox, Skelly \& John-Alder, 2003; Aguilar-Moreno et al., 2010). Lo que podría explicar el número total de puestas en $\mathrm{A}$. gularis (Vitt \& Breitenbach, 1993) y los procesos facultativos entre el número y tamaño de los huevos, similar a lo que sucede en Sceloporus undulatus (Vitt \& Breitenbach, 1993; Pianka \& Vitt, 2003). Por lo tanto, es importante considerar que los integrantes del clado sureste del complejo A. gularis y otros miembros del género, presentan expresiones fenotípicas altamente moldeables que les permiten enfrentar diferentes presiones de selección.

Es importante considerar la importancia de analizar cuantitativamente los efectos en la frecuencia reproductora y el tamaño de nidada, además de considerar que para los miembros del género Aspidoscelis, los efectos en la variación de historias de vida pueden responder a otras variables y mecanismos de selección. Sin embargo, en este trabajo solo se consideró la precipitación y sus posibles consecuencias. Lo que aporta al conocimiento ecológico y evolutivo en este grupo de vertebrados.

\section{AGRADECIMIENTOS}

Al American Museum of Natural History (AMNH) de Nueva York por el financiamiento otorgado para realizar el trabajo de campo para este trabajo. Al Centro de Investigaciones Biológicas de la UAEH por el permiso otorgado para el trabajo de laboratorio. A J. Eduardo González Espinoza por su ayuda 
en los procesos estadísticos y a Sara I. Valenzuela Ceballos por su apoyo y comentarios.

\section{RESUMEN}

Las historias de vida son atributos altamente variables que maximizan la adecuación de los organismos. Se ha documentado la relación del peso y el tamaño corporal sobre estos atributos, los cuales además estan influidos por variaciones ambientales y la heterogeneidad del hábitat. El tamaño y forma corporal son considerados atributos de historia de vida, los cuales estan principalmente asociados a la precipitación que promueve la cantidad de recursos disponibles en el ambiente. En el género Aspidoscelis el tamaño y masa relativa de la nidada estan asociados principalmente a la latitud y altitud. En Aspidoscelis gularis la precipitación de invierno favorece dos temporadas reproductoras, lo que determina que el tamaño de nidada sea variable en cada puesta. Las muestras fueron tomadas de mayo-julio 2013 y mayo-septiembre 2015. Se obtuvieron un total de 65 individuos de los cuales se tomaron medidas de longitud del cuerpo y distancia interaxilar, así como muestras de tejido hepático para la extracción de ADN y obtener las relaciones filogenéticas con respecto a un análisis de Inferencia Bayesiana para posteriormente aplicar los Métodos Comparados Filogenéticos como señal filogenética, contrastes filogenéticamente independientes y la reconstrucción del carácter ancestral. Por lo tanto, nuestros resultados muestran que en el tamaño y forma del cuerpo existe poca señal filogenética, en tanto que los contrastes filogenéticamente independientes y la reconstrucción del carácter ancestral sugieren que los tamaños corporales pequeños están asociadas a localidades con mayor cantidad de precipitación, lo que puede estar relacionado con un establecimiento de la madurez sexual temprana lo cual es el reflejo de la talla máxima de los adultos. Además, de acuerdo a un ANOVA y un ANCOVA hubo diferencias estadísticamente significativas en el tamaño y forma corporal respectivamente, lo que promueve un sistema para la competencia sexual para machos y un sistema para la fecundidad en hembras. Estos resultados son importantes para determinar el efecto de la precipitación sobre algunas características de historia de vida, lo que indica que las lagartijas del complejo sureste del complejo A. gularis son capaces de enfrentar diferentes presiones de selección, impuestas por el ambiente.

Palabras clave: método comparado filogenético, señal filogenética, contrastes filogenéticamente independientes, historias de vida, historia evolutiva.

\section{REFERENCIAS}

Aguilar-Moreno, M., Rodríguez-Romero, F. J., AragónMartínez, A., Muñoz-Manzano, J. A., GranadosGonzález, G., \& Hernández-Gallegos, O. (2010). Dimorfismo sexual de Aspidoscelis costata costata
(Squamata: Teiidae) en el sur del Estado De México, México. Revista Chilena de Historia Natural, 83(4), 585-592.

Angilleta, M. J., Oufiero, C. E., \& Leaché, A. D. (2006). Direct and indirect effects of environmental temperature on the evolution of reproductive strategies: an information-theoretic approach. The American Naturalist, 168(4), 123-135.

Ballinger, R. E. (1977). Reproductive strategies: food availability as a source of proximal variation in a lizard. Ecology, 58(3), 628-635.

Benabib, M. (2009). Los vertebrados y las historias de vida. En J. J. Morrone \& P. Magaña (Eds.), Evolución biológica (pp. 167-188). México: Universidad Nacional Autónoma de México.

Blomberg, S. P., Garland JR. T., \& Ives. A. R. (2003). Testing for phylogenetic signal in comparative data: behavioral traits are more labile. Evolution, 57(4), 717-745.

Blueweiss, L., Fox, H., Kudzma, V., Nakashima, D., Peters, R., \& Sams, S. (1978). Relationships between body size and some life history parameters. Oecologia, $37(2), 257-272$.

Brandt, R., \& Navas, C. A. (2011). Life-history evolution on Tropidurinae lizards: influence of lineage, body size and climate. Plos One, 6(5), E20040.

Chávez-García, M. (2010). Relaciones filogenéticas del complejo Aspidoscelis gularis (Sauria: Teiidae) en Cuatrociénegas, Coahuila (Tesis de Maestría sin publicar). Universidad Autónoma del Estado de Hidalgo, México.

Cox, R. M., Skelly, S. L., \& John-Alder, H. B. (2003). Comparative test of adaptive hypotheses for sexual size dimorphism in lizards. Evolution, 57(7), 1653-1669.

Espinosa, L. (2007). Guía práctica sobre la técnica de PCR. In. L. E. Eguiarte, V. Souza, \& X. Aguirre (Eds.), Ecología Molecular (pp. 517-540). México: SEMARNAT/IEUNAM/CONABIO.

Felsenstein, J. (1985). Phylogenies and the comparative method. American Naturalist, 125, 1-15.

Fetzner, J. W. (1999). Extracting high quality DNA from shed reptile skins: a simplified method. Biotechniques, 26(6), 1052-1054.

Grizante, M. B., Brandt, R., \& Kohlsdorf, T. (2012). Evolution of body elongation in Gymnophthalmid lizards: relationships with climate. Plos One, 7(11), E49772.

Harvey, P. H., \& Pagel, M. D. (1991). The comparative method in evolutionary biology. Oxford: Oxford University Press. 
Iraeta, P., Salvador, A., \& Díaz, J. A. (2012). Life-history traits of two mediterranean lizard populations: a possible example of countergradient covariation. Oecologia, 172(1), 167-176.

Maddison, W. P., \& Maddison, D. R. (2009). Mesquite: a modular system for evolutionary analysis (version 2.72). Disponible en: http://Mesquiteproject.Org. Acceso: 18 de Agosto de 2015.

Martins, E. P. (1996). Phylogenies, spatial autoregression, and the comparative method: a computer simulation test. Evolution, 50, 1750-1765.

Meiri, S., Yom-To, Y., \& Geffen, E. (2007). What determines conformity to Bergmann's rule? Global Ecology and Biogeography, 16, 788-794.

Mesquita, D. O., Gomes-Faria, R., Rinaldi-Colli, G., Vitt L. J., \& Pianka E. R., (2016). Lizard life-history strategies. Austral Ecology, 41(1), 1-5 doi: 10.1111/ aec. 12296

Michael, D. R., Banks, S. C., Piggott, M. P., Cunningham, R. B., Crane, M., Macgregor, C., Mcburney, C., \& Lindenmayer, D. B (2014). Geographical variation in body size and sexual size dimorphism in an australian lizard, Boulenger's skink (Morethia Boulengeri). Plos One, 9(10), E109830.

Midford, P. E, Garland JR, T., \& Maddison, W. P. (2010). Pdap:Pdtree: a translation of the Pdtree application of Garland et al., Phenotypic diversity analysis programs. Disponible en: http://Mesquiteproject.Org/ Pdap_Mesquite/Index.Html. Acceso: 28 de septiembre de 2015 .

Norma oficial mexicana NOM-062-ZOO-1999, Especificaciones técnicas para la producción, cuidado y uso de los animales de laboratorio. México, D. F.

Palumbi, S. R. (1996). PCR and molecular systematics. In D. Hillis, C. Moritz, \& B. Mable (Eds.), Molecular systematics (Second edition). Sinauer Press.

Pérez-Almazán, C., Balderas-Plata, M. A., ManríquezMorán, N. L., Madrigal-Uribe, D., \& Antonio-Némiga, X. (2014). Distribución potencial del complejo Aspidoscelis gularis (Squamata: Teiidae) en México. CienciaUAT, 9(1), 15-22.

Pianka, E. R. (1970). Comparative autoecology of the lizard Cnemidophorus tigris in diferent of its geographic range. Ecology, 51, 703-720.

Pianka, E. R., \& Vitt, L. J. (2003). Lizards: windows to the evolution of diversity. California: University of California Press.
Pincheira-Donoso, D., \& Meiri, S. (2013). An intercontinental analysis of climate-driven body size clines in reptiles: no support for patterns, no signals of processes. Evolutionary Biology, 40(4), 562-578.

R Core Team. (2011). R: a language and environment for statistical computing. $\mathrm{R}$ foundation for statistical computing. Vienna: Austria. ISBN 3-900051-07-0. Disponible en: http:/Www.R-Project.Org. Acesso: 19 de Octubre de 2015.

Roitberg, E. G., Eplanova, V., Kotenko, T. I., Amat, F., Carretero. M. A., Kuranova, V. N., Bulakhova, N. A., Zinenko, O. I., \& Yakovlev, V. A. (2015). Geographic variation of life-history traits in the sand lizard Lacerta agilis: testing Darwin's fecundityadvantage hypothesis. Journal of Evolutionary Biology, 28, 613-629.

Shine, R. (1980). "Costs" of reproduction in reptiles. Oecologia, 46, 92-100.

Shine, R. (1989). Ecological causes for the evolution of sexual dimorphism: a review of the evidence. Quarterly Review of Biology, 64, 419-461.

Stearns, S. (1976). Life history tactics: a review of the ideas. Quarterly Review of Biology, 51(1), 3-47.

Stearns, S. (1992). The evolution of life histories. Oxford: Oxford University Press.

Tamura, K., Stecher, G., Peterson, D., Filipski, A., \& Kumar, S. (2013). MEGA6: Molecular Evolutionary Genetics Analysis version 6.0. Molecular Biology and Evolution, 12, 2725-9.

Turner, F. B., Medica, P. A., \& Smith, D. D. (1974). Reproduction and survivorship of the lizard, Uta Stansburiana, and the effects of winter rainfall, density and predation on these processes. U.S. International Biological Program, Desert Biome, Utah State University, Logan, Utah, Reports of 1973. Progress, Volume 3: Process studies, Rm 74-26.

Vitt, L. J., \& Breitenbach, G. L. (1993). Life histories and reproductive tactics among lizards in the genus Cnemidophorus (Sauria: Teiidae). In J. W. Wright, \& L. J. Vitt (Eds.), Biology of whiptail lizards (Genus Cnemidophorus) (pp. 211-244). Oklahoma: Oklahoma Museum of Natural History.

Zamora-Abrego, G., Manríquez-Morán, N. L., Ortíz-Yusty, C. E., \& Ortega-León, A. M. (2013). Uso de técnicas moleculares como herramienta para conservar la diversidad biológica. In A. López-Herrera (Ed.), Biología molecular aplicada a la producción animal y la conservación de especies silvestres (pp. 313-386). Colombia: Universidad Nacional De Colombia. 
